\title{
Double-Spin Leukocyte-Rich Platelet-Rich Plasma Is Predominantly Lymphocyte Rich With Notable Concentrations of Other White Blood Cell Subtypes
}

\author{
Anuj Marathe, B.B.A., Shiv J. Patel, B.S., Bo Song, M.D., Joseph M. Sliepka, M.D., \\ Theodore S. Shybut, M.D., Brendan H. Lee, M.D., Ph.D., and Prathap Jayaram, M.D.
}

\begin{abstract}
Purpose: To comprehensively characterize a double-spin leukocyte-rich platelet-rich plasma (LR-PRP) formulation and to compare it with whole blood (WB) by quantitatively assessing platelet and WB cell subtype concentrations in each. Methods: Prospective human ex vivo analysis with 12 healthy adult men with ages ranging from 25 to 31 was performed in a controlled laboratory setting. The main outcome measure was the leukocyte profile of human LR-PRP. Results: In LR-PRP, lymphocytes were the predominant WB cell type $\left(11.94 \pm 2.97 \times 10^{3}\right.$ cells/ $\left.\mu \mathrm{L}\right)$ followed by neutrophils $\left(3.72 \pm 1.28 \times 10^{3}\right.$ cells $\left./ \mu \mathrm{L}\right)$. The mean cumulative percentage of granulocytes was $23 \% \pm 8 \%$ and agranulocytes was $77 \% \pm 18 \%$. There was a significant difference observed between granulocyte and agranulocyte percentage within both WB $(P=.004,[95 \%$ CI: $(7 \%, 31 \%)])$ and LR-PRP $(P<.0001,[95 \%$ CI: $(42 \%, 66 \%)])$ groups. In addition, there was a significant difference observed between the WB and LR-PRP granulocyte percentages $(P<.0001,[95 \%$ CI: $(29 \%, 43 \%)])$ and between the WB and LR-PRP agranulocyte percentages $(P<.0001,[95 \%$ CI: (30\%,42\%)]). Conclusions: Our study found that LR-PRP is predominantly lymphocyte rich with notable concentrations of other WB cell subtypes, including neutrophils, monocytes, eosinophils, basophils, and large unstained cells. While these subtypes are not routinely reported, they may play a role in modulating the local inflammatory environment. We also found significant differences in WB cell subtype concentrations between WB and LR-PRP. Clinical Relevance: PRP has been routinely used in many clinical practices without clear indications for its use and lacks standardization in its formulation. This study provides a comprehensive characterization of a broadly used PRP, LR-PRP, and further characterizes subtypes of WBC cells present in LR-PRP that have not been previously reported. Comprehensively reporting these subtypes in clinical trials of PRP is crucial to understanding how these cells participate in PRP's therapeutic potential. This type of data can help standardize future PRP formulations and improve patient outcomes.
\end{abstract}

\section{Introduction}

A utologous cell therapies, including platelet-rich plasma (PRP), have become more prevalent in recent years for the treatment of musculoskeletal injuries and soft tissue diseases, such as lateral epicondylitis, knee osteoarthritis, rotator cuff injury, and more. ${ }^{1}$ PRP is

H. Ben Taub Dept of Physical Medicine $\theta$ Rehabilitation, Baylor College of Medicine, Houston, Texas, U.S.A. (A.M., S.J.P., B.S., J.M.S., P.J.); Department of Molecular and Human Genetics, Baylor College of Medicine, Houston, Texas, U.S.A. (B.H.L.); Texas Children's Hospital, Houston, Texas, U.S.A. (T.S.S.); Department of Orthopedic Surgery, Baylor College of Medicine, Houston, Texas, U.S.A. (T.S.S.); and Department of Physical Medicine and Rehabilitation and Orthopedic Surgery, Regenerative Sports Medicine, Baylor College of Medicine, Houston, Texas, U.S.A. (P.J.).

The authors report the following potential conflicts of interest or sources of funding: The authors have received funding from the BCM Intellectual and Developmental Disabilities Research Center (HD024064) from the Eunice Kennedy Shriver National Institute of Child Health and Human Development, the BCM Advanced Technology Cores with funding from the National Institutes of Health (CA125123), the Rolanette and Berdon Lawrence Bone Disease Program of Texas, the BCM Center for Skeletal Medicine and Biology, defined as the processed liquid fraction of autologous peripheral blood, which has a platelet concentration above the baseline. ${ }^{1}$ It has been established, mainly through preclinical data, that PRP's therapeutic impact is likely due to the abundant source of growth factors and signaling molecules in PRP. ${ }^{2}$ Clinically, there have been

the Pamela and David Ott Center for Heritable Disorders of Connective Tissue, and the Richard S. Materson Education Research Grant through the PMR Foundation. Full ICMJE author disclosure forms are available for this article online, as supplementary material.

Received June 30, 2021; accepted October 4, 2021.

Address correspondence to Prathap Jayaram, M.D., H. Ben Taub Department of Physical Medicine and Rehabilitation, 7200 Cambridge St., Suite 10C, Houston, TX 77030.E-mail: jayaram@bcm.edu

(C) 2021 THE AUTHORS. Published by Elsevier Inc. on behalf of the Arthroscopy Association of North America. This is an open access article under the CC BY-NC-ND license (http://creativecommons.org/licenses/by-nc-nd/4.0/). 2666-061X/21914

https://doi.org/10.1016/j.asmr.2021.10.004 
34 randomized control trials demonstrating PRP's clinical benefit in knee osteoarthritis. ${ }^{3}$ Despite these encouraging trials, there is significant heterogeneity in reporting PRP formulations within these trials. ${ }^{3}$ Currently, PRP has been classified into two broad groups: leukocyte-rich PRP (LR-PRP) and leukocyte-poor PRP (LP-PRP). Furthermore, LR-PRP has not been further delineated among its various cell subtypes.

There are two distinct PRP preparation methods. The first, often employed intraoperatively, uses continuousflow centrifuge bowl or disk separation techniques and employs standard blood cell separators on autologously harvested blood. ${ }^{1}$ The second is used more in the point-ofcare setting and uses gravitational centrifugation to isolate the buffy coat layer. ${ }^{1}$ In brief, PRP is prepared by drawing blood from the patient and centrifuging to separate its components. Then, the supernatant is aspirated along with the platelet-containing buffy coat layer and homogenized (single-spin) or put through another spin (double-spin). If a double-spin protocol is used, the platelets will pellet after the second spin. They can then be homogenized with the upper or lower halves of the supernatant to form leukocyte-poor and leukocyte-rich PRP, respectively. After the PRP is isolated, it can be activated exogenously using calcium chloride, autologous thrombin, type 1 collagen, or photo activation. It can also be immediately injected into the site of interest for endogenous activation. ${ }^{4,5}$ Activation causes platelet degranulation, releasing growth factors that have important downstream effects in restoring an environment for optimal healing potential. ${ }^{2,5}$

Historically, there has been little standardization and consensus in the formulation of PRP products. As such, it is possible that patients may have varying responses to these products on the basis of their individual biological profiles. While recent studies have made improvements to the general classification of PRP with regard to platelets, there is a lack of research studying the effects that white blood cells (WBCs) have on the healing process. Even most classification systems only report the presence or absence of WBCs in PRP without delineating specific WBC subtypes. Given their unique cell contributions that affect local healing and regeneration, comprehensively reporting WBC subtypes in clinical trials of PRP is crucial to understanding how these cells participate in PRP's therapeutic potential. This type of data can help standardize future PRP formulations and improve patient outcomes. The purposes of this study were to comprehensively characterize a double-spin LR-PRP formulation and to compare it to $\mathrm{WB}$ by quantitatively assessing platelet and $\mathrm{WB}$ cell subtype concentrations in each. We hypothesized that LR-PRP's predominant subtype would primarily be neutrophil rich compared to baseline whole blood.

\section{Methods}

\section{Study Population}

The research protocol in this study was approved by the institutional review board at the institution where the research was conducted (IRB protocol H-44684). Healthy study participants were identified during clinic visits. Exclusion criteria were the following: age less than 18 or greater than 35 , known comorbidities or chronic disease, and history of tobacco, alcohol, or substance use. Normal leukogram and platelet count profile was required for inclusion. On the basis of these criteria, 12 healthy adult men from ages 25 to 31 were included. The rationale behind these criteria was intentional in order to sample a more homogenous PRP and avoid confounding from gender, age, chronic disease, and substance use-associated factors.

\section{Experimental Protocol}

Blood draw was conducted at rest without the patient having taken any medications. $30 \mathrm{~mL}$ of whole blood (WB) was drawn from each participant into a syringe containing $3 \mathrm{~mL}$ of citrate dextrose anticoagulant solution, yielding a total volume of $33 \mathrm{~mL} .75 \mathrm{~mL}$ was used to obtain a complete blood count (CBC), and the remaining $32.25 \mathrm{~mL}$ was used to prepare the LR-PRP. We followed the manufacturer's double-spin protocol for preparing the LR-PRP (Smart-Prep PC-30; Harvest Terumo BCT). The first spin lasts for 1-3 minutes at a speed of 2,500 $\pm 150 \mathrm{rpm}$, and the second lasts for 6-9 minutes at a speed of 2,300 $\pm 150 \mathrm{rpm}$. This system yields a final LR-PRP volume of $\sim 4 \mathrm{~mL}$. After obtaining the LR-PRP, a CBC was obtained, and platelet concentrations were verified using scatter cytogram and platelet-integrated analysis (ADVIA 120 Hematology Analyzer, Siemens). ${ }^{6}$ In terms of WBC differential classification, granulocytes consist of neutrophils, eosinophils, and basophils, while agranulocytes consist of lymphocytes, monocytes, and large unstained cells (LUCs).

Analysis was carried out in the form of descriptive statistics, paired sample $t$-tests, and ANOVA using GraphPad Prism and Microsoft Excel. Results are reported as means \pm SD. Differences among groups were evaluated with a 2-tailed ratio paired $t$-tests along with a reflex analysis of effective pairing. Differences were accepted as statistically significant at $P<.05[95 \% \mathrm{CI}]$.

\section{Power Analysis}

Sample size was determined for the primary outcome of assessing WBC subtypes in LR-PRP compared to baseline whole blood. The null hypothesis is that there is no difference in the primary outcome between LRPRP and WB. The alternative hypothesis is that there is a difference in the primary outcome between LR-PRP and WB. Sample size estimations were done on the basis of statistical significance found in controlled laboratory settings. ${ }^{6}$ 


\section{Results}

\section{Analysis}

Our study population consisted of 12 , healthy adult male volunteers, aged 25 to 31 . The mean age of the participants was $27.91 \pm 3.12$ years.

For the $12 \mathrm{WB}$ samples collected, the mean platelet concentration was $196.25 \pm 33.70 \times 10^{3}$ platelets $/ \mu \mathrm{L}$. For the 12 PRP samples, the mean platelet concentration was $1,391.67 \pm 275.07 \times 10^{3}$ platelets/ $\mu \mathrm{L}$ (Fig 1). This represents an $\sim 7.09$-fold increase in platelet concentration from WB to LR-PRP $(P<.0001$, [95\% CI: $(1039,1351)]$.

The paired sample $t$-tests between WB and LR-PRP groups demonstrated significant differences between total white blood cells (WBCs) cells $(P<.0001,[95 \%$ CI: $(10.74,14.73)])$, neutrophil $(P=.001,[95 \%$ CI: $(.59,1.72)])$, basophil $(P<.0001$, [95\% CI: $(.12, .21)]$, lymphocyte $(P<.0001$, [95\% CI: $(8.70,11.94)]$, monocyte $(P<.0001$, [95\% CI: $(.62,1.02)]$, and LUC $(P<.0001$, [95\% CI: $(.26, .38)]$ concentrations. Eosinophil concentrations were not significantly different [95\% CI: $(-.08, .005)]$.

\section{Leukocyte Profile}

In WB, neutrophils were the predominant WBC type $\left(2.56 \pm .68 \times 10^{3}\right.$ cells $\left./ \mu \mathrm{L}\right)$ followed by lymphocytes $\left(1.62 \pm .45 \times 10^{3}\right.$ cells $\left./ \mu \mathrm{L}\right)$. In LR-PRP, lymphocytes were the predominant WBC type $\left(11.94 \pm 2.97 \times 10^{3}\right.$ cells $/ \mu \mathrm{L})$ followed by neutrophils $\left(3.72 \pm 1.28 \times 10^{3}\right.$ cells $/ \mu \mathrm{L})$. The data from the leukocyte profile of the WB and LR-PRP samples are summarized in Table 1. The WBC makeup of WB and LR-PRP are represented graphically in Fig 2.

\section{Granulocytes Versus Agranulocytes}

In WB, the mean cumulative percentage of granulocytes was $60 \% \pm 15 \%$, and the mean cumulative percentage of agranulocytes was $40 \% \pm 10 \%$. In LRPRP, the mean cumulative percentage of granulocytes was $23 \% \pm 8 \%$ and agranulocytes was $77 \% \pm 18 \%$ (Fig 3). There was a significant difference observed between granulocyte and agranulocyte percentage within both WB $(P=.004,[95 \%$ CI: $(7 \%, 31 \%)])$ and LR-PRP $(P<.0001$, [95\% CI: $(42 \%, 66 \%)])$ groups. In addition, there was a significant difference observed between the WB and LR-PRP granulocyte percentages $(P<.0001$, [95\% CI: $(29 \%, 43 \%)])$ and between the WB and LR-PRP agranulocyte percentages $(P<.0001$, [95\% CI: $(30 \%, 42 \%)])$.

\section{Discussion}

The data from our study demonstrate that LR-PRP is predominantly lymphocyte rich with notable concentrations of other WBC subtypes. There are also significant differences in WB cell subtype concentrations between WB and LR-PRP. Although these subtypes are not routinely reported in clinical trials, they may play a role in modulating the local inflammatory environment. In trials that do report WBC subtypes for PRP, there is an emphasis on neutrophils being the predominant cell type, ${ }^{2}$ which differs from our results, which showed a lymphocyte-predominant subtype in LR-PRP. Our findings also show that the predominant WBC subtype changes from neutrophils in WB to lymphocytes in LR-PRP (Fig 2). Still, studies continue to broadly categorize their leukocyte content without providing complete subtype characterization. For example, even within the 34 clinical trials of PRP in knee OA, LR-PRP's characterization, with the exception of one trial by $\mathrm{Wu}$ et al., ${ }^{7}$ is limited to total leukocyte content alone. ${ }^{3}$ Even the few clinical trials that use an established classification system will typically only report on the neutrophil and/or lymphocyte content for their formulation. ${ }^{7-10}$ In fact, current classification systems, such as the PAW, PLRA, DEPA, and MARSPILL, do not include a comprehensive description of the WBC subtypes in PRP. ${ }^{11}$ In this study, we were able to delineate the other WBC subtypes found in LR-PRP in addition to neutrophils and lymphocytes. We found that all cell types, except eosinophils, had higher concentrations in LR-PRP than in WB (Table 1). Additionally, when compared to WB, LR-PRP had a significantly lower percentage of granulocytes and a significantly higher percentage of agranulocytes. Physiologically, granulocytes from the myeloid stem cell line function mostly within the innate immune system and mediate the initial inflammatory response at the site of infection or injury, while agranulocytes function mostly within the adaptive immune system. ${ }^{12}$ These specific

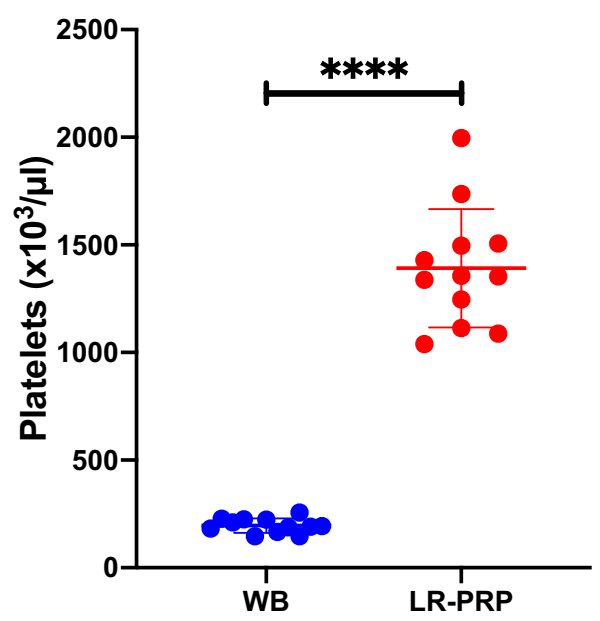

Fig 1. Platelet concentration reported in $10^{3} / \mu \mathrm{L}$ in whole blood (WB) and leukocyte-rich platelet rich plasma (LR-PRP). Concentrations were obtained using a scatter cytogram and the platelet-integrated analysis method. Participants in each group: $n=12$. 
Table 1. WBC and WBC Components in Whole Blood and LR-PRP

\begin{tabular}{lccc}
\hline & Whole Blood $\left(10^{3}\right.$ cells $\left./ \mu \mathrm{L}\right)$ & LR-PRP $\left(10^{3}\right.$ cells $\left./ \mu \mathrm{L}\right)$ & Concentration Ratio $(\mathrm{LR}-\mathrm{PRP}: \mathrm{WB})$ \\
\hline Total WBCs & $4.57 \pm .80$ & $17.31 \pm 3.65$ & $3.79 \pm .58$ \\
Neutrophils & $2.56 \pm .68$ & $3.72 \pm 1.28$ & $1.45 \pm .34$ \\
Eosinophils & $.14 \pm .06$ & $.10 \pm .06$ & $.74 \pm .53$ \\
Basophils & $.03 \pm .01$ & $.19 \pm .08$ & $6.68 \pm 4.00$ \\
Lymphocytes & $1.62 \pm .45$ & $11.94 \pm 2.97$ & $7.36 \pm .64$ \\
Monocytes & $.19 \pm .04$ & $1.02 \pm .35$ & $5.34 \pm 1.13$ \\
LUCs & $.03 \pm .01$ & $0.34 \pm .10$ & $12.15 \pm 6.77$
\end{tabular}

Leukocyte profile of WB samples $(n=12)$ and LR-PRP samples $(n=12)$ are summarized in Table 1. Cell concentrations are reported in units of $10^{3}$ cells $/ \mu \mathrm{L}$ and reported as means \pm SD. LR-PRP:WB, leukocyte rich platelet rich plasma:whole blood; LUC, large unsustained cells; WBC, white blood cell.

subtype differences in WBC function underscore the importance of comprehensive reporting of PRP formulations in clinical trials.

\section{White Blood Cells}

WBCs (leukocytes) are generally categorized into neutrophils, monocytes, lymphocytes, eosinophils, basophils, and large unsustained cells (LUC). ${ }^{12}$ Leukocytes originate from either myeloid or lymphoid stem cells. Myeloid stem cells give rise to neutrophils, eosinophils, basophils, and monocytes, which give rise to macrophages. Lymphoid stem cells give rise to lymphocytes, which include $\mathrm{B}$ cells, $\mathrm{T}$ cells, and natural killer cells. Leukocytes, due to their function in the immune and host-defense mechanisms, play a large role in the immune modulation of tissue repair. ${ }^{13}$ As a result, we believe they may influence the effects of PRP. Despite this, some PRP protocols call for a deliberate reduction of leukocytes to levels below baseline, also known as leukocyte-poor PRP (LP-PRP). Although it has been suggested that leukocytes may be proinflammatory and detrimental to joints, ${ }^{14}$ there are other studies that have directly compared LR-PRP and LPPRP. In fact, Mariani et al. found that LR-PRP did not produce a proinflammatory response in vivo. ${ }^{15}$ In addition, Yaradilmis et al. found that LR-PRP produced more obvious improvements in visual analog scale and Western Ontario and McMaster Universities Osteoarthritis Index scores at 2, 6, and 12 months in moderategrade gonarthrosis compared to LP-PRP. ${ }^{10}$ These studies further demonstrate the complexity of the interaction between WBCs and tissue healing and the need for more thorough research to directly compare the two formulations. By rigorously documenting WBC subtypes, future studies can set the stage for large data analysis of the positive and negative effects that individual WBC subtypes have on their local environment. This may allow for better individualized optimization of cell content in PRP formulations for patients.

\section{Neutrophils}

Neutrophils are the most prevalent WBC and usually constitute between 60 and $70 \%$ of leukocytes in WB. ${ }^{12}$ They are most commonly associated with the inflammatory response and defend against bacterial and fungal infections. Neutrophils also serve other proinflammatory functions and are thought to promote catabolic effects through the release of inflammatory cytokines and matrix metalloproteinases. ${ }^{16}$ Researchers even found that PRP rich in neutrophils could create a higher collagen type III to type I ratio, leading to fibrosis and reduced tendon integrity. ${ }^{17}$
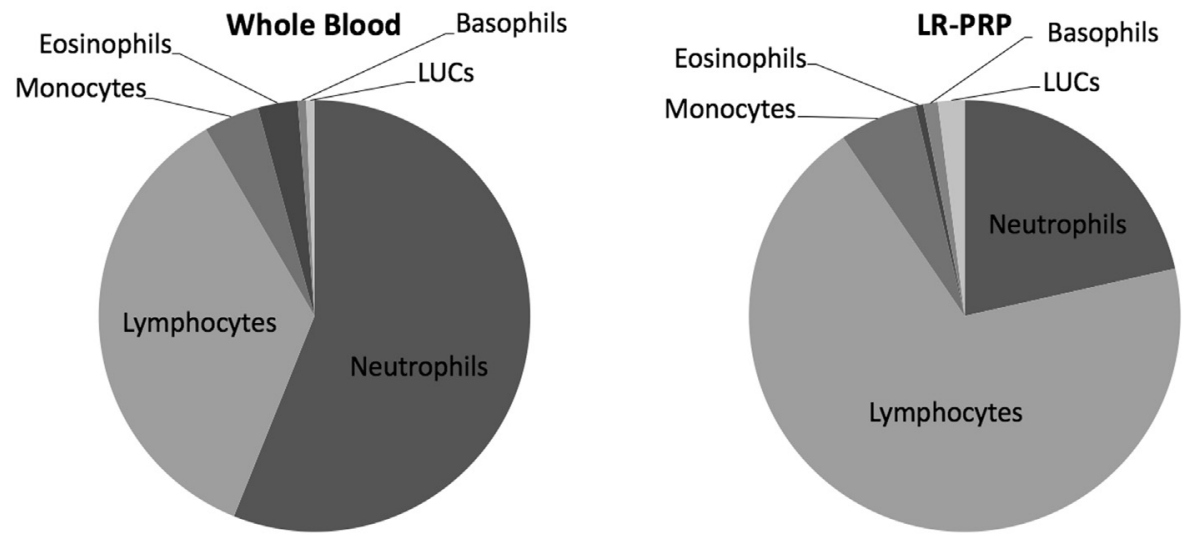

Fig 2. Pie chart representations of white blood cell components in whole blood and leukocyte-rich platelet rich plasma (LR-PRP). LUCs, large unsustained cells. 
Mean Percentage Granulocyte vs. Agranulocyte

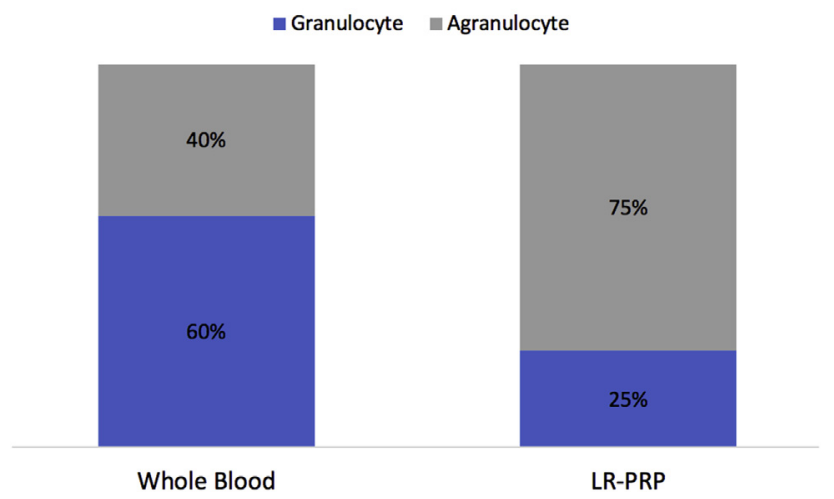

Fig 3. Stacked comparison of mean percentage of granulocytes and agranulocytes in whole blood and leukocyte-rich platelet rich plasma (LR-PRP).

On the other hand, there is also evidence to support the role of neutrophils in tissue healing. In fact, neutrophils may function to promote muscle growth and tissue regeneration after disease or injury through IL-6 activation of satellite cells. ${ }^{18}$ IL-6 can induce a regenerative response by activating satellite cells to proliferate, fusing, and repairing damaged muscle fibers in a mechanism similar to embryonic myogenesis. ${ }^{18}$ Another pathway in which neutrophils mediate a more anti-inflammatory response is by converting arachidonic acid to leukotriene A4, which is converted by activated platelets into lipoxin A4, a potent antiinflammatory protein capable of inhibiting the proinflammatory neutrophil response. ${ }^{19,20}$ Marwick et al. also demonstrated that neutrophils could induce monocyte/macrophage anti-inflammatory reprogramming, which would aid in resolution of acute inflammation and local healing and repair. ${ }^{21}$

\section{Eosinophils}

Eosinophils are quite rare in circulation, constituting 1-6\% of total WBCs and known mainly for their response in allergic reactions and parasitic infections. ${ }^{12}$ However, evidence points to their integral role in the inflammatory response, immunomodulation, and even healing. ${ }^{22}$ They secrete and have receptors for a whole host of proinflammatory cytokines, as well as MHC-II receptors allowing them to serve as antigenpresenting cells. This allows them to modulate T-helper cell differentiation. ${ }^{22}$ Interestingly, Heredia et al. found that IL-4-secreting eosinophils were integral to muscle regeneration by signaling local stem cell differentiation. ${ }^{23}$ Additionally, loss of eosinophils in vivo was shown to severely compromise muscle regeneration. ${ }^{23}$

\section{Basophils}

Basophils are the least prevalent WBC, constituting less than $.5 \%$ of total WBCs. ${ }^{12}$ They most notably respond to allergens and antigens by releasing chemicals that result in the dilation of blood vessels. Because of their relative scarcity, less is known about their function in tissue repair. They are thought to contribute to the multifaceted process of angiogenesis and lymphangiogenesis, primarily in the context of tumor production. ${ }^{24}$

\section{Large Unstained Cells}

Modern cell analyzers, including the one used in this study, are able to differentiate leukocytes based on their peroxidase activity. Neutrophils, monocytes, and eosinophils exhibit peroxidase activity and are, thus, able to be stained. However, lymphocytes exhibit no peroxidase activity and are unstained. ${ }^{25}$ Thus, large unstained cells (LUCs) are cells that cannot be classified as lymphocytes (normal or otherwise) or stem cells and exhibit no peroxidase activity. ${ }^{26}$ Currently, there are no clinical studies that explore the purpose of these cells as it relates to inflammation or tissue repair.

\section{Lymphocytes}

Lymphocytes account for around $30 \%$ of WBCs in WB and give rise to B cells, helper cells, and cytotoxic $\mathrm{T}$ cells, and natural killer cells. ${ }^{12}$ Although these cells are crucial in cell-mediated adaptive immunity against pathogens, they play a more indirect role in the tissue healing process. They accomplish this by steering monocyte differentiation toward M2 anti-inflammatory subtype. ${ }^{27,28}$

\section{Monocytes}

Monocytes are the largest leukocytes, constitute around $5 \%$ of the WBCs in WB, and serve as phagocytes in the primary immune response. ${ }^{12}$ They also have an active role in wound healing, as macrophage dysfunction is commonly seen in nonhealing and poorly healed wounds. ${ }^{29}$ In addition, they stimulate angiogenesis and fibroplasia. ${ }^{30}$ During homeostasis and differentiation, monocytes can differentiate into two main phenotypes of macrophages. The first is proinflammatory Ml, whose main purpose is to kill pathogens. ${ }^{31}$ The second is the anti-inflammatory M2, which aids in tissue repair. ${ }^{32}$ This M2 class has 3 known subtypes, M2a, M2b, and M2c. M2a produces extracellular matrix, collagen, and growth factors to drive wound closure. ${ }^{31}$ M2b and M2c work to suppress inflammation through interleukin-10 (IL-10) production. ${ }^{31,32}$

It is possible that these monocytes populations have increased functionality under the conditions created by platelet degranulation in PRP. For example, it is well documented that platelet granules release a multitude of growth factors including large quantities of platelet factor 4 (PF4). ${ }^{33} \mathrm{PF} 4$ is crucial for monocyte chemotaxis to injured tissue and prevents monocyte apoptosis in vitro. ${ }^{34}$ In addition to PF4, activated platelets were 
found to selectively release PGE2, which led to increased levels of IL-10 and suppressed tumor necrosis factor- $\alpha$, creating an anti-inflammatory environment. ${ }^{35}$ Finally, a recent study by Lee et al. demonstrated that activated platelets skewed monocyte differentiation to the M2 subset and can even convert some monocytes from M1 to M2. ${ }^{36}$ Put together, it is clear that the monocytes found in PRP have the potential to significantly modulate the inflammatory environment at the site of local injection.

On the basis of our results, it is clear that LR-PRP is a heterogeneous mixture of not only platelets but also many different WBC types that can contribute to the healing process. Despite this fact, studies do not report the full composition of LR-PRP formulations. Without data to compare patient outcomes and cell concentrations, it is difficult to delineate which of the WBCs participate in modulating many of the dysregulated processes in musculoskeletal diseases. One contributing effort to bridge this gap would be to comprehensively quantify all WBC subtypes in clinical trials moving forward. This could enable future studies to link patient outcome metrics to cellular compositions in PRP, which could then be translated into more mechanistically driven research.

\section{Limitations}

Our study was limited by its relatively small sample size and inability to characterize whether there is a difference for PRP between age or gender. With respect to the latter, while healthy men between the ages 25 and 31 are not representative of a population that PRP would normally be used in, our goal was to characterize WBC subtypes in a healthy and homogenous population, free of any confounding variables from other potential chronic illnesses.

\section{Conclusion}

Our study found that LR-PRP is predominantly lymphocyte rich with notable concentrations of other WBC subtypes, including neutrophils, monocytes, eosinophils, basophils, and LUCs. Although these subtypes are not routinely reported, they may play a role in modulating the local inflammatory environment. We also found significant differences in WBC subtype concentrations between WB and LR-PRP.

\section{References}

1. Everts P, Onishi K, Jayaram P, Lana JF, Mautner K. Plateletrich plasma: New performance Understandings and therapeutic considerations in 2020. IJMS 2020;21:7794.

2. Andia I, Maffulli N. Platelet-rich plasma for managing pain and inflammation in osteoarthritis. Nat Rev Rheumatol 2013;9:721-730.

3. Filardo G, Previtali D, Napoli F, Candrian C, Zaffagnini S, Grassi A. PRP injections for the treatment of knee osteoarthritis: A meta-analysis of randomized controlled trials. Cartilage. In press, https://doi.org/10.1177/ 1947603520931170.

4. Paterson KL, Nicholls M, Bennell KL, Bates D. Intraarticular injection of photo-activated platelet-rich plasma in patients with knee osteoarthritis: A double-blind, randomized controlled pilot study. BMC Musculoskelet Disord $2016 ; 17: 67$.

5. Cavallo C, Roffi A, Grigolo B, et al. Platelet-rich plasma: The choice of activation method affects the release of bioactive molecules. BioMed Res Int 2016;2016:1-7.

6. Jayaram P, Yeh P, Patel SJ, et al. Effects of aspirin on growth factor release from freshly isolated leukocyte-rich platelet-rich plasma in healthy men: A prospective fixedsequence controlled laboratory study. Am J Sports Med 2019;47:1223-1229.

7. Wu Y-T, Hsu K-C, Li T-Y, Chang C-K, Chen L-C. Effects of platelet-rich plasma on pain and muscle strength in patients with knee osteoarthritis. Am J Phys Med Rehabil 2018;97:248-254.

8. Gobbi A, Lad D, Karnatzikos G. The effects of repeated intra-articular PRP injections on clinical outcomes of early osteoarthritis of the knee. Knee Surg Sports Traumatol Arthrosc 2015;23:2170-2177.

9. Huang Y, Liu X, Xu X, Liu J. Intra-articular injections of platelet-rich plasma, hyaluronic acid or corticosteroids for knee osteoarthritis: A prospective randomized controlled study. Orthopäde 2019;48:239-247.

10. Yaradilmis YU, Demirkale I, Safa Tagral A, Caner Okkaoglu M, Ates A, Altay M. Comparison of two platelet rich plasma formulations with viscosupplementation in treatment of moderate grade gonarthrosis: A prospective randomized controlled study. J Orthopaed 2020;20: 240-246.

11. Rossi LA, Murray IR, Chu CR, Muschler GF, Rodeo SA, Piuzzi NS. Classification systems for platelet-rich plasma. Bone Joint J 2019;101-B:891-896.

12. Handin R, Lux S, Stossel T. Blood: principles and practice of hematology. Philidelphia, PA: Lippincott Williams \& Wilkins, 2003.

13. Leoni G, Neumann P-A, Sumagin R, Denning TL, Nusrat A. Wound repair: Role of immune-epithelial interactions. Mucosal Immunol 2015;8:959-968.

14. Braun HJ, Kim HJ, Chu CR, Dragoo JL. The effect of platelet-rich plasma formulations and blood products on human synoviocytes: Implications for intra-articular injury and therapy. Am J Sports Med 2014;42: 1204-1210.

15. Mariani E, Canella V, Cattini L, et al. Leukocyte-rich platelet-rich plasma injections do not up-modulate intraarticular pro-inflammatory cytokines in the osteoarthritic knee. PLOS ONE 2016;11:e0156137.

16. Fedorova NV, Ksenofontov AL, Serebryakova MV, et al. Neutrophils release metalloproteinases during adhesion in the presence of insulin, but cathepsin G in the presence of glucagon. Mediators Inflamm 2018;2018:1-9.

17. Zhou Y, Wang JH-C. PRP treatment efficacy for tendinopathy: A review of basic science studies. BioMed Res Int 2016;2016:1-8.

18. Toumi H, F'guyer S, Best TM. The role of neutrophils in injury and repair following muscle stretch. J Anat 2006;208:459-470. 
19. Parrish WR. Physiology of blood components in wound healing: An appreciation of cellular co-operativity in platelet-rich plasma action. JESO 2017;4:1-14.

20. Lana JF, Macedo A, Ingrao ILG, Huber SC, Santos GS, Santana MHA. Leukocyte-rich PRP for knee osteoarthritis: Current concepts. J Clin Orthopaed Trauma 2019;10: S179-S182.

21. Marwick JA, Mills R, Kay O, et al. Neutrophils induce macrophage anti-inflammatory reprogramming by suppressing NF-KB activation. Cell Death Dis 2018;9:665.

22. Jacobsen EA, Helmers RA, Lee JJ, Lee NA. The expanding role(s) of eosinophils in health and disease. Blood 2012;120:3882-3890.

23. Heredia JE, Mukundan L, Chen FM, et al. Type 2 innate signals stimulate fibro/adipogenic progenitors to facilitate muscle regeneration. Cell 2013;153:376-388.

24. Marone G, Varricchi G, Loffredo S, Granata F. Mast cells and basophils in inflammatory and tumor angiogenesis and lymphangiogenesis. Eur J Pharmacol 2016;778:146-151.

25. Bononi A, Lanza F, Dabusti M, et al. Increased myeloperoxidase index and large unstained cell values can predict the neutropenia phase of cancer patients treated with standard dose chemotherapy. Cytometry 2001;46:92-97.

26. Thirup P. LUC, What is that? Clin Chem 1999;45:1 100-1100.

27. Tiemessen MM, Jagger AL, Evans HG, van Herwijnen MJC, John S, Taams LS. CD $4^{+} \mathrm{CD} 25^{+}$Foxp $^{+}$ regulatory $\mathrm{T}$ cells induce alternative activation of human monocytes/macrophages. Proc Natl Acad Sci 2007;104 (19),446-19,451.
28. Weirather J, Hofmann UDW, Beyersdorf N, et al. Foxp3 ${ }^{+}$ $\mathrm{CD} 4{ }^{+} \mathrm{T}$ cells improve healing after myocardial infarction by modulating monocyte/macrophage differentiation. Circ Res 2014;115:55-67.

29. Koh TJ, DiPietro LA. Inflammation and wound healing: the role of the macrophage. Expert Rev Mol Med 201 1;13:e23.

30. Reinke JM, Sorg H. Wound repair and regeneration. Eur Surg Res 2012;49:35-43.

31. Mosser DM, Edwards JP. Exploring the full spectrum of macrophage activation. Nat Rev Immunol 2008;8:958-969. doi:10.1038/nri2448.

32. Ferrante CJ, Leibovich SJ. Regulation of macrophage polarization and wound healing. Adv Wound Care 2012;1: $10-16$.

33. Lakka Klement G, Shai E, Varon D. The role of platelets in angiogenesis In: Platelets. New York: Elsevier, 2013;487-502.

34. Schaffner A. Regulated expression of platelet factor 4 in human monocytes-Role of PARs as a quantitatively important monocyte activation pathway. J Leukoc Biol 2005;78:202-209.

35. Linke B, Schreiber Y, Picard-Willems B, et al. Activated platelets induce an anti-inflammatory response of monocytes/macrophages through cross-regulation of $\mathrm{PGE}_{2}$ and cytokines. Mediat Inflamm 2017;2017:1-14.

36. Lee SJ, Yoon BR, Kim HY, Yoo S-J, Kang SW, Lee W-W. Activated platelets convert $\mathrm{CD}_{1} 4^{+} \mathrm{CD} 16-$ into $\mathrm{CD} 14^{+} \mathrm{CD}_{16} 6^{+}$monocytes with enhanced Fc $\gamma \mathrm{R}$-mediated phagocytosis and skewed M2 polarization. Front Immunol 2021;11:611133. 